\title{
EXTENDED BLOCKER, DELETION, AND CONTRACTION MAPS ON ANTICHAINS
}

\author{
ANDREY O. MATVEEV
}

Received 18 June 2002

\begin{abstract}
Families of maps on the lattice of all antichains of a finite bounded poset that extend the blocker, deletion, and contraction maps on clutters are considered. Influence of the parameters of the maps is investigated. Order-theoretic extensions of some principal relations for the set-theoretic blocker, deletion, and contraction maps on clutters are presented.
\end{abstract}

2000 Mathematics Subject Classification: 06A06, 90C27.

1. Introduction and preliminary. Let $P$ be a finite bounded poset of cardinality greater than one. We can define some maps on the lattice of all antichains $\mathcal{A}(P)$ of the poset $P$ that naturally extend the set-theoretic blocker, deletion, and contraction maps on clutters; such maps were considered in $[4,5]$.

A set $H$ is called a blocking set for a nonempty family $\varphi=\left\{G_{1}, \ldots, G_{m}\right\}$ of nonempty subsets of a finite set if, for each $k \in\{1, \ldots, m\}$, it holds $\left|H \cap G_{k}\right|>0$. The family of all inclusionwise minimal blocking sets for $\varphi$ is called the blocker of $\mathscr{G}$. We denote the blocker of $\mathscr{G}$ by $\mathscr{B}(\mathscr{G})$.

A family of subsets of a finite ground set $S$ is called a clutter or a Sperner family if no set from that family contains another. The empty clutter $\varnothing$ containing no subsets of $S$ and the clutter $\{\hat{0}\}$ whose unique set is the empty subset 0 of $S$ are called the trivial clutters on $S$. The set-theoretic blocker map reflects a nontrivial clutter to its blocker, and that map reflects a trivial clutter to the other trivial clutter: $\mathscr{B}(\varnothing)=\{\hat{0}\}$ and $\mathscr{B}(\{\hat{0}\})=\varnothing$.

Let $X \subseteq S$ and $|X|>0$. The set-theoretic deletion $(\backslash X)$ and contraction $(/ X)$ maps are defined in the following way: if $\varphi$ is a nontrivial clutter on $S$, then the deletion $\mathscr{G} \backslash X$ is the family $\{G \in \mathscr{G}:|G \cap X|=0\}$ and the contraction $\mathscr{G} / X$ is the family of all inclusionwise minimal sets from the family $\{G-X: G \in \mathscr{G}\}$. The deletion and contraction for the trivial clutters coincide with the clutters $\varnothing \backslash X=\varnothing / X=\varnothing$ and $\{\hat{0}\} \backslash X=\{\hat{0}\} / X=\{\hat{0}\}$. The maps $(\backslash \hat{0})$ and $(/ \hat{0})$ are the identity map on clutters; for any $\operatorname{clutter} \mathscr{G}$, we by definition have $\mathscr{G} \backslash \hat{0}=\mathscr{G} / \hat{0}=\mathscr{G}$.

Let $\varphi$ be a clutter on the ground set $S$. Given a subset $X \subseteq S$, we have

$$
\begin{gathered}
\mathscr{B}(\mathscr{B}(\mathscr{G}))=\mathscr{G}, \\
\mathscr{B}(\mathscr{G}) \backslash X=\mathscr{B}(\mathscr{G} / X), \quad \mathscr{B}(\mathscr{G}) / X=\mathscr{B}(\mathscr{G} \backslash X) .
\end{gathered}
$$


Recall that the atoms of the poset $P$ are the elements covering its least element. Let $X$ be a subset of the atom set $P^{\text {a }}$ of $P$. (We denote the empty subset of $P^{\mathrm{a}}$ by $\varnothing^{\mathrm{a}}$.) We use the denotation $\boldsymbol{6}: \mathcal{A}(P) \rightarrow \mathcal{A}(P)$ for the order-theoretic blocker map from [4], and we use the denotations $(\backslash X),(/ X): \mathcal{A}(P) \rightarrow \mathcal{A}(P)$ for the order-theoretic operators of deletion and contraction from [5], respectively. We do not recall those concepts here because the map $b$ is the $\left(\varnothing^{\mathrm{a}}, 0\right)$-blocker map from Definition 2.1 of the present paper and the maps $(\backslash X)$ and $(/ X)$ are the $(X, 0)$-deletion and $(X, 0)$-contraction maps from Definition 3.1 of the present paper, respectively.

For any antichain $A$ of $P$, the following relations hold in $\mathcal{A}(P)$ :

$$
\begin{gathered}
\mathfrak{b}(\mathfrak{b}(\mathfrak{b}(A)))=\mathfrak{b}(A), \\
\mathfrak{b}(A) \backslash X \leq \mathfrak{b}(A / X) \leq \mathfrak{b}(A) \leq \mathfrak{b}(A) / X \leq \mathfrak{b}(A \backslash X) .
\end{gathered}
$$

Equality (1.3) from [4] goes back to (1.1) from [2, 3]. Comparison (1.4) from [5] goes back to (1.2) from [6].

In the present paper, we consider families of the so-called $(X, k)$-blocker, $(X, k)$-deletion, and $(X, k)$-contraction maps on $\mathcal{H}(P)$ parametrized by subsets $X \subseteq P^{\mathrm{a}}$ and numbers $k \in \mathbb{N}, k<\left|P^{\mathrm{a}}\right|$. We show that for all pairs of the abovementioned parameters $X$ and $k$, the essential properties of the maps remain similar to those of the $\left(\varnothing^{\mathrm{a}}, 0\right)$-blocker, $(X, 0)$-deletion, and $(X, 0)$-contraction maps on $\mathcal{A}(P)$ that were investigated in $[4,5]$. In particular, we present analogues of relations (1.3) and (1.4) in Proposition 2.6(ii) and Theorem 3.7.

We refer the reader to [7, Chapter 3] for basic information and terminology in the theory of posets.

We use $\min Q$ to denote the set of all minimal elements of a poset $Q$. If $Q$ has a least element, then it is denoted $\hat{0}_{Q}$; if $Q$ has a greatest element, then it is denoted $\hat{1}_{Q}$.

Throughout the paper, $P$ stands for a finite bounded poset of cardinality greater than one, that is, $P$ by definition has the least and greatest elements that are distinct. We denote by $\mathfrak{I}(A)$ and $\mathfrak{f}(A)$ the order ideal and filter of $P$ generated by an antichain $A$, respectively.

All antichains of $P$ compose a distributive lattice denoted $\mathcal{A}(P)$; in the present paper, antichains are by definition partially ordered in the following way; if $A^{\prime}, A^{\prime \prime} \in \mathcal{H}(P)$, then we set

$$
A^{\prime} \leq A^{\prime \prime} \quad \text { iff } \mathfrak{f}\left(A^{\prime}\right) \subseteq \mathfrak{f}\left(A^{\prime \prime}\right)
$$

We call the least and greatest elements $\hat{0}_{\mathcal{A}(P)}$ and $\hat{1}_{\mathcal{A}(P)}$ of $\mathcal{A}(P)$ the trivial antichains of $P$ because, in the context of the present paper, they are counterparts of the trivial clutters. Here, $\hat{O}_{\mathfrak{A}(P)}$ is the empty antichain of $P$ and $\hat{1}_{\mathfrak{A}(P)}$ the oneelement antichain $\left\{\hat{0}_{P}\right\}$. We denote by $\vee$ and $\wedge$ the operations of join and meet 
in the lattice $\mathcal{A}(P)$; if $A^{\prime}, A^{\prime \prime} \in \mathcal{H}(P)$, then

$$
\begin{aligned}
& A^{\prime} \vee A^{\prime \prime}=\min \left(A^{\prime} \cup A^{\prime \prime}\right), \\
& A^{\prime} \wedge A^{\prime \prime}=\min \left(\mathfrak{f}\left(A^{\prime}\right) \cap \mathfrak{f}\left(A^{\prime \prime}\right)\right) .
\end{aligned}
$$

2. $(X, k)$-blocker map. In this section, we consider a family of maps on antichains of a finite bounded poset that extend the set-theoretic blocker map on clutters. From now on, $X$ is always a subset of $P^{\mathrm{a}}$ and $k$ is a nonnegative integer less than $\left|P^{\mathrm{a}}\right|$.

Definition 2.1. The $(X, k)$-blocker map on $\mathcal{A}(P)$ is the map $\mathfrak{b}_{k}^{X}: \mathfrak{A}(P) \rightarrow$ A $(P)$,

$$
A \longmapsto \min \left\{b \in P:\left|\mathfrak{I}(b) \cap \mathfrak{I}(a) \cap\left(P^{\mathrm{a}}-X\right)\right|>k \forall a \in A\right\}
$$

if $A$ is nontrivial, and

$$
\hat{0}_{\mathfrak{A}(P)} \longmapsto \hat{1}_{\mathfrak{A}(P)}, \quad \hat{1}_{\mathfrak{H}(P)} \longmapsto \hat{0}_{\mathfrak{A}(P)} .
$$

Given an antichain $A \in \mathcal{A}(P)$, the antichain $\mathfrak{6}_{k}^{X}(A)$ is the $(X, k)$-blocker of $A$ in $P$.

We use the denotations $\mathfrak{b}_{k}$ and $\mathfrak{b}^{X}$ instead of the denotations $\mathfrak{b}_{k}^{\varnothing^{\mathrm{a}}}$ and $\mathfrak{b}_{0}^{X}$, respectively. The $\left(\varnothing^{\mathrm{a}}, 0\right)$-blocker map is the blocker map $\mathfrak{b}$ on $\mathcal{A}(P)$ considered in [4]. Given $A \in \mathcal{H}(P)$, the antichain $\mathfrak{b}(A)$ is called the blocker of $A$ in $P$.

If $\{a\}$ is a one-element antichain of $P$, then we write $\mathfrak{b}_{k}^{X}(a)$ instead of $\mathfrak{b}_{k}^{X}(\{a\})$. Let $a \neq \hat{0}_{P}$. Since the blocker map on $\mathcal{A}(P)$ is antitone, for every $E \subseteq \mathfrak{b}(a)-X$, we have $\{a\} \leq \mathfrak{b}(\mathfrak{b}(a)) \leq \mathfrak{b}(\mathfrak{b}(a)-X) \leq \mathfrak{b}(E) \leq \mathfrak{b}(a)$.

The following statement immediately follows from Definition 2.1.

LEMMA 2.2. Let $A$ be a nontrivial antichain of $P$. If $\mathfrak{b}_{k}^{X}(A) \neq \hat{O}_{\mathfrak{A}(P)}$, then, for each $a \in A$ and for all $b \in \mathfrak{b}_{k}^{X}(A)$, it holds that

$$
\left|\mathfrak{I}(a) \cap \mathfrak{I}(b) \cap\left(P^{\mathrm{a}}-X\right)\right|>k .
$$

Let $a \in P, a \neq \hat{0}_{P}$. From now on, $\mathscr{T}_{a}$ denotes the family of subsets of the atom set $P^{\mathrm{a}}$ defined as follows:

$$
\mathscr{T}_{a}=\{E \subseteq \mathfrak{b}(a)-X:|E|=k+1\} .
$$

Let $\mathcal{L}\left(P^{\mathrm{a}}\right)$ denote the Boolean lattice of all subsets of the atom set $P^{\mathrm{a}}$, and let $\mathcal{L}\left(P^{\mathrm{a}}\right)^{(k+1)}$ denote the subset of all elements of rank $k+1$ of $\mathcal{L}\left(P^{\mathrm{a}}\right)$. Given a $(k+1)$-subset $E \subseteq P^{\mathrm{a}}$, we denote by $\varepsilon(E)$ the least upper bound for $E$ in $\mathcal{L}\left(P^{\mathrm{a}}\right)$; conversely, given an element $e \in \mathcal{L}\left(P^{\mathrm{a}}\right)^{(k+1)}$, we denote by $\varepsilon^{-1}(\mathfrak{e})$ the $(k+1)$ subset of all atoms of $\mathcal{L}\left(P^{\mathrm{a}}\right)$ that are comparable with $e$. 
Let $A$ be a nontrivial antichain of $P$. If $|\mathfrak{b}(a)-X| \leq k$ for some $a \in A$, then Definition 2.1 implies $\mathfrak{b}_{k}^{X}(A)=\hat{0}_{\mathcal{H}(P)}$. In the case $|\mathfrak{b}(a)-X|>k$ for all $a \in A$, Proposition 2.3 describes two alternative ways of elementwise finding the $(X, k)$-blocker of $A$; it involves the set-theoretic blocker $\mathscr{B}(\cdot)$ of a set family.

Proposition 2.3. Let $A$ be a nontrivial antichain of $P$. If $|\mathfrak{b}(a)-X|>k$, for all $a \in A$, then

$$
\mathfrak{b}_{k}^{X}(A)=\bigwedge_{a \in A} \bigvee_{E \in \mathscr{T}_{a}} \mathfrak{b}(E)=\bigvee_{\in \in \mathscr{B}\left(\left\{\left\{\varepsilon(E): E \in \mathcal{T}_{a}\right\}: a \in A\right\}\right)} \bigwedge_{\mathfrak{e} \in \mathfrak{E}} \mathfrak{b}\left(\varepsilon^{-1}(\mathfrak{e})\right)
$$

Proof. We have

$$
\mathfrak{b}_{k}^{X}(A)=\bigwedge_{a \in A} \mathfrak{b}_{k}^{X}(a)
$$

and an order-theoretic argument shows that, for every $a \in A$, it holds that

$$
\mathfrak{b}_{k}^{X}(a)=\bigvee_{E \in \mathscr{T}_{a}} \mathfrak{b}(E)
$$

where $\mathfrak{b}(E)=\bigwedge_{e \in E}\{e\}$.

The inclusion $\mathfrak{b}_{k}^{X}(A) \supseteq \bigvee_{\mathfrak{E} \in \mathscr{B}\left(\left\{\left\{\varepsilon(E): E \in \mathcal{T}_{a}\right\}: a \in A\right\}\right)} \bigwedge_{\mathfrak{e} \in \mathfrak{E}} \mathfrak{b}\left(\varepsilon^{-1}(\mathfrak{e})\right)$ follows from Definition 2.1. To prove the inclusion

$$
\mathfrak{b}_{k}^{X}(A) \subseteq \bigvee_{\in \in \mathscr{B}\left(\left\{\left\{\varepsilon(E): E \in \mathcal{T}_{a}\right\}: a \in A\right\}\right)} \bigwedge_{\mathfrak{e} \in \mathfrak{E}} \mathfrak{b}\left(\varepsilon^{-1}(\mathfrak{e})\right),
$$

assume that it does not hold. Consider an element $b \in \mathfrak{b}_{k}^{X}(A)$ such that it does not belong to the right-hand side of (2.8). In this case, there is an element $a \in A$ such that $\left|\mathfrak{I}(b) \cap \mathfrak{I}(a) \cap\left(P^{\mathrm{a}}-X\right)\right| \leq k$. It means that the left-hand side of (2.8) is not an $(X, k)$-blocker of $A$, a contradiction.

The following lemma clarifies how the parameters of the $(X, k)$-blocker map influence the image of $\mathfrak{A}(P)$; additionally, the lemma states that $\mathfrak{b}_{k}^{X}$ is antitone.

LEMMA 2.4. (i) Let $Y \subseteq P^{\mathrm{a}}, Y \supseteq X$, and let $j$ be a nonnegative integer, $j \leq k$. If $A \in \mathcal{A}(P)$, then

$$
\mathfrak{b}_{j}^{X}(A) \geq \mathfrak{b}_{k}^{X}(A) \geq \mathfrak{b}_{k}^{Y}(A)
$$

(ii) For all $A^{\prime}, A^{\prime \prime} \in \mathcal{H}(P)$ such that $A^{\prime} \leq A^{\prime \prime}$, it holds that

$$
\mathfrak{b}_{k}^{X}\left(A^{\prime}\right) \geq \mathfrak{b}_{k}^{X}\left(A^{\prime \prime}\right)
$$


Proof. (i) There is nothing to prove if $A$ is trivial. Suppose that $A$ is a nontrivial antichain of $P$. For each element $a \in A$, we by (2.7) have

$$
\mathfrak{b}_{k}^{X}(a)=\bigvee_{E \in \mathcal{T}_{a}} \mathfrak{b}(E) \geq \bigvee_{\substack{E \subseteq \mathfrak{b}(a)-Y: \\|E|=k+1}} \mathfrak{b}(E)=\mathfrak{b}_{k}^{Y}(a)
$$

With respect to (2.6), this yields

$$
\mathfrak{b}_{k}^{X}(A)=\bigwedge_{a \in A} \mathfrak{b}_{k}^{X}(a) \geq \bigwedge_{a \in A} \mathfrak{b}_{k}^{Y}(a)=\mathfrak{b}_{k}^{Y}(A)
$$

The relation $\mathfrak{b}_{j}^{X}(A) \geq \mathfrak{b}_{k}^{X}(A)$ is proved in a similar way.

(ii) If $A^{\prime}$ is a trivial antichain, then the assertion immediately follows from Definition 2.1. Suppose that $A^{\prime}$ is nontrivial. For every $a^{\prime} \in A^{\prime}$, there is $a^{\prime \prime} \in A^{\prime \prime}$ such that $\left\{a^{\prime}\right\} \leq\left\{a^{\prime \prime}\right\}$ and, as a consequence, it holds the inclusion $\mathfrak{b}\left(a^{\prime}\right) \supseteq$ $\mathfrak{b}\left(a^{\prime \prime}\right),(2.7)$ implies $\mathfrak{b}_{k}^{X}\left(a^{\prime}\right) \geq \mathfrak{b}_{k}^{X}\left(a^{\prime \prime}\right)$, and the proof is completed by applying (2.6).

In addition to Lemma 2.4(ii), we need the following statement to describe the structure of the image of $\mathcal{A}(P)$ under the $(X, k)$-blocker map.

LEMMA 2.5. For any $A \in \mathfrak{A}(P)$, it holds that

$$
\mathfrak{6}_{k}^{X}\left(\mathfrak{b}_{k}^{X}(A)\right) \geq A
$$

Proof. If $A$ is a trivial antichain of $P$, then the lemma follows from Definition 2.1 because, in this case, we have $\mathfrak{b}_{k}^{X}\left(\mathfrak{b}_{k}^{X}(A)\right)=A$. Suppose that $A$ is nontrivial. If $\mathfrak{b}_{k}^{X}(A)=\hat{0}_{\mathfrak{A}(P)}$, then we have $\mathfrak{b}_{k}^{X}\left(\mathfrak{b}_{k}^{X}(A)\right)=\hat{1}_{\mathfrak{A}(P)} \geq A$ and we are done. Finally, suppose that $b_{k}^{X}(A)$ is a nontrivial antichain. On the one hand, according to Lemma 2.2, for each $a \in A$ and for all $b \in \mathfrak{b}_{k}^{X}(A)$, it holds that

$$
\left|\mathfrak{Z}(a) \cap \mathfrak{I}(b) \cap\left(P^{\mathrm{a}}-X\right)\right|>k .
$$

On the other hand, we, by Definition 2.1, have

$$
\mathfrak{b}_{k}^{X}\left(\mathfrak{G}_{k}^{X}(A)\right)=\min \left\{g \in P:\left|\mathfrak{z}(g) \cap \mathfrak{I}(b) \cap\left(P^{\mathrm{a}}-X\right)\right|>k \forall b \in \mathfrak{b}_{k}^{X}(A)\right\} .
$$

Hence, we have $\mathfrak{b}_{k}^{X}\left(\mathfrak{b}_{k}^{X}(A)\right) \geq A$.

We complete this section by applying a standard technique of the theory of posets to the lattice $\mathcal{A}(P)$ and the $(X, k)$-blocker map on it. See, for instance, $[1$, Chapter IV] on (co)closure operators. 
Proposition 2.6. (i) The composite map $\mathfrak{b}_{k}^{X} \circ \mathfrak{b}_{k}^{X}$ is a closure operator on $\mathcal{A}(P)$.

(ii) The poset $\mathfrak{B b}_{k}^{X}(P)=\left\{\mathfrak{b}_{k}^{X}(A): A \in \mathfrak{A}(P)\right\}$ is a self-dual lattice; the restriction map $\left.\mathfrak{b}_{k}^{X}\right|_{\mathfrak{B}_{k}^{X}(P)}$ is an anti-automorphism of $2_{k}^{X}(P)$. The lattice $2_{k}^{X}(P)$ is a meetsubsemilattice of the lattice $\mathcal{A}(P)$.

(iii) For every $B \in \mathfrak{B}_{k}^{X}(P)$, its preimage $\left(\mathfrak{b}_{k}^{X}\right)^{-1}(B)$ under the $(X, k)$-blocker map is a convex join-subsemilattice of the lattice $\mathcal{A}(P)$. The greatest element of $\left(\mathfrak{b}_{k}^{X}\right)^{-1}(B)$ is $\mathfrak{b}_{k}^{X}(B)$.

PROOF. In view of Lemmas 2.4(ii) and 2.5, assertions (i) and (ii) are a corollary of [1, Propositions 4.36 and 4.26]. To prove (iii), choose arbitrary elements $A^{\prime}, A^{\prime \prime} \in\left(\mathfrak{G}_{k}^{X}\right)^{-1}(B)$, where $B=\mathfrak{b}_{k}^{X}(A)$ for some $A \in \mathcal{A}(P)$, and note that $\mathfrak{b}_{k}^{X}\left(A^{\prime} \vee A^{\prime \prime}\right)=\mathfrak{b}_{k}^{X}\left(A^{\prime}\right) \wedge \mathfrak{b}_{k}^{X}\left(A^{\prime \prime}\right)=B$. If $B=\hat{0}_{\mathcal{H}(P)}$, then $\mathfrak{b}_{k}^{X}(B)=\hat{1}_{\mathfrak{A}(P)}$ is the greatest element of $\left(\hat{b}_{k}^{X}\right)^{-1}(B)$. If $B=\hat{1}_{\mathcal{A}(P)}$, then $\left(\mathfrak{b}_{k}^{X}\right)^{-1}(B)$ is the one-element subposet $\left\{\hat{O}_{\mathcal{H}(P)}\right\}$ of $\mathcal{A}(P)$. Finally, if $B$ is a nontrivial antichain of $P$, then the element $\mathfrak{b}_{k}^{X}(B)=\mathfrak{b}_{k}^{X}\left(\mathfrak{b}_{k}^{X}(A)\right)$ is by (2.15) the greatest element of $\left(\mathfrak{b}_{k}^{X}\right)^{-1}(B)$. Since the $(X, k)$-blocker map is antitone, we can see that the subposet $\left(6_{k}^{X}\right)^{-1}(B)$ of $\mathcal{A}(P)$ is convex.

We call the poset $23_{k}^{X}(P)$ from Proposition 2.6(ii) the lattice of $(X, k)$-blockers in $P$. The poset $\mathfrak{2}(P)=\mathfrak{2}_{0}^{\varnothing^{\mathrm{a}}}(P)$ is called in [4] the lattice of blockers in $P$.

3. $(X, k)$-deletion and $(X, k)$-contraction maps. In this section, we consider order-theoretic extensions of the set-theoretic deletion and contraction maps on clutters.

DEFINITION 3.1. (i) If $\{a\}$ is a nontrivial one-element antichain of $P$, then the $(X, k)$-deletion $\{a\} \backslash_{k} X$ and $(X, k)$-contraction $\{a\} /_{k} X$ of $\{a\}$ in $P$ are the antichains

$$
\begin{aligned}
& \{a\} \backslash_{k} X= \begin{cases}\{a\}, & \text { if }|\mathfrak{b}(a) \cap X| \leq k, \\
\hat{\mathfrak{O}}_{\mathfrak{A}(P)}, & \text { if }|\mathfrak{b}(a) \cap X|>k,\end{cases} \\
& \{a\} /_{k} X= \begin{cases}\{a\}, & \text { if }|\mathfrak{b}(a) \cap X| \leq k, \\
\mathfrak{b}_{k}^{X}\left(\mathfrak{b}_{k}^{X}(a)\right), & \text { if }|\mathfrak{b}(a) \cap X|>k, \mathfrak{b}(a) \nsubseteq X, \\
\hat{1}_{\mathfrak{A}(P)}, & \text { if }|\mathfrak{b}(a) \cap X|>k, \mathfrak{b}(a) \subseteq X .\end{cases}
\end{aligned}
$$

(ii) If $A$ is a nontrivial antichain of $P$, then the $(X, k)$-deletion $A \backslash_{k} X$ and $(X, k)$-contraction $A / k X$ of $A$ in $P$ are the antichains

$$
A \backslash_{k} X=\bigvee_{a \in A}\left(\{a\} \backslash_{k} X\right), \quad A /{ }_{k} X=\bigvee_{a \in A}\left(\{a\} /{ }_{k} X\right)
$$


(iii) The $(X, k)$-deletion and $(X, k)$-contraction of the trivial antichains of $P$ are

$$
\begin{aligned}
& \hat{0}_{\mathfrak{A}(P)} \backslash_{k} X=\hat{0}_{\mathfrak{H}(P)} / /_{k} X=\hat{0}_{\mathfrak{H}(P)} \text {, } \\
& \hat{1}_{\mathfrak{A}(P)} \backslash_{k} X=\hat{1}_{\mathfrak{A}(P)} / /_{k} X=\hat{1}_{\mathcal{H}(P)} \text {. }
\end{aligned}
$$

(iv) The map

$$
\left(\backslash_{k} X\right): \mathcal{A}(P) \longrightarrow \mathcal{A}(P), \quad A \longmapsto A \backslash_{k} X
$$

is the operator of $(X, k)$-deletion on $\mathcal{A}(P)$.

The map

$$
(/ k X): \mathcal{H}(P) \longrightarrow \mathcal{H}(P), \quad A \longmapsto A /{ }_{k} X,
$$

is the operator of $(X, k)$-contraction on $\mathcal{H}(P)$.

Given an antichain $A \in \mathcal{H}(P)$, we use the denotations $A \backslash X$ and $A / X$ instead of the denotations $A \backslash_{0} X$ and $A /{ }_{0} X$, respectively. The $(X, 0)$-deletion map $(\backslash X): \mathcal{A}(P) \rightarrow \mathcal{A}(P)$ and the $(X, 0)$-contraction map $(/ X): \mathcal{A}(P) \rightarrow \mathcal{A}(P)$ are the operators of deletion and contraction on $\mathcal{A}(P)$, respectively, considered in [5].

The following observation is an immediate consequence of Definition 3.1. If $a^{\prime}, a^{\prime \prime} \in P$ and $\left\{a^{\prime}\right\} \leq\left\{a^{\prime \prime}\right\}$ in $\mathcal{A}(P)$, then

$$
\left\{a^{\prime}\right\} \backslash_{k} X \leq\left\{a^{\prime \prime}\right\} \backslash_{k} X, \quad\left\{a^{\prime}\right\} /_{k} X \leq\left\{a^{\prime \prime}\right\} /_{k} X
$$

hence, in view of (3.3) and (3.4), we can formulate the following lemma.

LEMMA 3.2. If $A^{\prime}, A^{\prime \prime} \in \mathcal{H}(P)$ and $A^{\prime} \leq A^{\prime \prime}$, then

$$
A^{\prime} \backslash_{k} X \leq A^{\prime \prime} \backslash_{k} X, \quad A^{\prime} /_{k} X \leq A^{\prime \prime} /_{k} X
$$

Moreover, if $\{a\}$ is a one-element antichain of $P$, then we have

$$
\{a\} \backslash_{k} X \leq\{a\} \leq\{a\} / /_{k} X
$$

and a more general statement is true.

LEMMA 3.3. If $A \in \mathfrak{A}(P)$, then

$$
A \backslash_{k} X \leq A \leq A / k_{k} X
$$

Another consequence of Definition 3.1 is that, for a one-element antichain $\{a\}$ of $P$, it holds that

$$
\mathfrak{b}_{k}^{X}(a) \backslash_{k} X \leq \mathfrak{b}_{k}^{X}\left(\{a\} /_{k} X\right) \leq \mathfrak{b}_{k}^{X}(a) \leq \mathfrak{b}_{k}^{X}(a) /_{k} X \leq \mathfrak{b}_{k}^{X}\left(\{a\} \backslash_{k} X\right) .
$$


Let $\{a\}$ be a nontrivial one-element antichain of $P$. We obviously have $\left(\{a\} \backslash_{k} X\right) \backslash_{k} X=\{a\} \backslash_{k} X$. We show that $\left(\{a\} /{ }_{k} X\right) /_{k} X=\{a\} /{ }_{k} X$. If $|\mathfrak{b}(a) \cap X| \leq k$, then Definition 3.1 implies $\left(\{a\} /{ }_{k} X\right) / k_{k} X=\{a\} / k X=\{a\}$; further, if $|\mathfrak{b}(a) \cap X|>$ $k$ and $\boldsymbol{b}(a) \subseteq X$, then Definition 3.1 implies $\left(\{a\} /_{k} X\right) /_{k} X=\{a\} / k_{k} X=\hat{1}_{\mathcal{H}(P)}$. Suppose that $|\mathfrak{b}(a) \cap X|>k$ and $\mathfrak{b}(a) \nsubseteq X$. In this case, on the one hand, we have $\left(\{a\} / k_{k} X\right) /_{k} X \geq\{a\} / k_{k} X$ by Lemma 3.3, on the other hand, for every element $b \in\{a\} /{ }_{k} X=\mathfrak{b}_{k}^{X}\left(\mathfrak{b}_{k}^{X}(a)\right)$, we have $\mathfrak{b}_{k}^{X}(b) \geq \mathfrak{b}_{k}^{X}(a)$, and, as a consequence, we have $\left(\{a\} /_{k} X\right) /_{k} X=\bigvee_{b \in\{a\} /_{k} X}\left(\{b\} /{ }_{k} X\right) \leq \mathfrak{b}_{k}^{X}\left(\mathfrak{b}_{k}^{X}(a)\right)=\{a\} /_{k} X$. We arrive at the conclusion that $\left(\{a\} / k_{k} X\right) /_{k} X=\{a\} / k X$. With respect to (3.3), we can formulate the following lemma.

LEMMA 3.4. If $A \in \mathcal{A}(P)$, then

$$
\left(A \backslash_{k} X\right) \backslash_{k} X=A \backslash_{k} X, \quad\left(A /{ }_{k} X\right) /_{k} X=A /{ }_{k} X .
$$

Lemmas 3.2, 3.3, and 3.4 lead to a characterization of the $(X, k)$-deletion and $(X, k)$-contraction maps in terms of (co)closure operators.

Proposition 3.5. The map $\left(\backslash_{k} X\right)$ is a coclosure operator on $\mathcal{A}(P)$. The map $\left(/ /_{k} X\right)$ is a closure operator on $\mathfrak{A}(P)$.

The following proposition is a counterpart of Lemma 2.4(i).

Proposition 3.6. Let $Y \subseteq P^{\mathrm{a}}, Y \supseteq X$, and let $m$ be an integer, $k \leq m<\left|P^{\mathrm{a}}\right|$. If $A \in \mathcal{A}(P)$, then

$$
\begin{gathered}
A \backslash_{m} X \geq A \backslash_{k} X \geq A \backslash_{k} Y, \\
A /_{k} X \leq A /{ }_{k} Y \leq A / m Y .
\end{gathered}
$$

Proof. If $A$ is a trivial antichain, then the proposition follows from (3.4). Suppose that $A$ is nontrivial. For each $a \in A$, (3.1) implies $\{a\} \backslash_{k} X \geq\{a\} \backslash_{k} Y$, (3.2) implies $\{a\} / k X \leq\{a\} / k Y$, and (3.3) yields

$$
\begin{aligned}
& A \backslash_{k} X=\bigvee_{a \in A}\left(\{a\} \backslash_{k} X\right) \geq \bigvee_{a \in A}\left(\{a\} \backslash_{k} Y\right)=A \backslash_{k} Y, \\
& A /{ }_{k} X=\bigvee_{a \in A}\left(\{a\} /_{k} X\right) \leq \bigvee_{a \in A}\left(\{a\} /_{k} Y\right)=A /_{k} Y .
\end{aligned}
$$

Other relations are proved in a similar way.

We denote the images $\left(\backslash_{k} X\right)(\mathcal{A}(P))=\left\{A \backslash_{k} X: A \in \mathcal{A}(P)\right\}$ and $\left(/_{k} X\right)(\mathcal{H}(P))=$ $\left\{A /{ }_{k} X: A \in \mathcal{H}(P)\right\}$ by $\mathcal{H}(P) \backslash_{k} X$ and $\mathcal{H}(P) /_{k} X$, respectively. We can interpret well-known properties of (semi)lattice maps and (co)closure operators on lattices in the case of the $(X, k)$-deletion and $(X, k)$-contraction maps. 
Definition 3.1 implies that the maps $\left(\backslash_{k} X\right),\left(/_{k} X\right): \mathcal{A}(P) \rightarrow \mathcal{H}(P)$ are upper $\left\{\hat{0}_{\mathfrak{A}(P)}, \hat{1}_{\mathcal{H}(P)}\right\}$-homomorphisms, that is, for all $A^{\prime}, A^{\prime \prime} \in \mathcal{H}(P)$, we have $\left(A^{\prime} \vee\right.$ $\left.A^{\prime \prime}\right) \backslash_{k} X=\left(A^{\prime} \backslash_{k} X\right) \vee\left(A^{\prime \prime} \backslash_{k} X\right)$ and $\left(A^{\prime} \vee A^{\prime \prime}\right) /_{k} X=\left(A^{\prime} /_{k} X\right) \vee\left(A^{\prime \prime} /{ }_{k} X\right)$, and, moreover, we have $\hat{0}_{\mathfrak{A}(P)} \backslash_{k} X=\hat{0}_{\mathfrak{A}(P)} /{ }_{k} X=\hat{0}_{\mathfrak{A}(P)}$ and $\hat{1}_{\mathfrak{A}(P)} \backslash_{k} X=\hat{1}_{\mathfrak{A}(P) / k} X=\hat{1}_{\mathfrak{A}(P)}$.

The posets $\mathcal{H}(P) \backslash_{k} X$ and $\mathcal{A}(P) /_{k} X$, with the partial orders induced by the partial order on $\mathcal{H}(P)$, are lattices.

We call the poset $\mathcal{H}(P) \backslash_{k} X$ the lattice of $(X, k)$-deletions in $P$, and we call the poset $\mathcal{A}(P) /_{k} X$ the lattice of $(X, k)$-contractions in $P$.

The lattice $\mathfrak{A}(P) \backslash_{k} X$ is a join-subsemilattice of $\mathfrak{A}(P)$. Denote by $\wedge_{\mathcal{H}}(P) \backslash_{k} X$ the operation of meet in $\mathcal{A}(P) \backslash_{k} X$. If $D^{\prime}, D^{\prime \prime} \in \mathcal{H}(P) \backslash_{k} X$, then we have $D^{\prime} \wedge \mathcal{H}(P) \backslash_{k} X$ $D^{\prime \prime}=\left(D^{\prime} \wedge D^{\prime \prime}\right) \backslash_{k} X$.

The lattice $\mathcal{A}(P) /{ }_{k} X$ is a sublattice of $\mathcal{A}(P)$.

If $D \in \mathcal{H}(P) \backslash_{k} X$, then the preimage $\left(\backslash_{k} X\right)^{-1}(D)$ of $D$ under the $(X, k)$-deletion map is the closed interval $\left[D, D \vee \bigvee_{E \subseteq X:|E|=k+1} \mathfrak{b}(E)\right]$ of $\mathcal{A}(P)$.

If $D \in \mathcal{A}(P) /{ }_{k} X$, then the preimage $\left(/_{k} X\right)^{-1}(D)$ of $D$ under the $(X, k)$ contraction map is a convex join-subsemilattice of the lattice $\mathcal{A}(P)$, with the greatest element $D$.

Relations (1.2) and (1.4) have the following analogue.

THEOREM 3.7. If $A \in \mathfrak{A}(P)$, then

$$
\mathfrak{b}_{k}^{X}(A) \backslash_{k} X \leq \mathfrak{b}_{k}^{X}\left(A /_{k} X\right) \leq \mathfrak{b}_{k}^{X}(A) \leq \mathfrak{b}_{k}^{X}(A) /{ }_{k} X \leq \mathfrak{b}_{k}^{X}\left(A \backslash_{k} X\right) .
$$

Proof. There is nothing to prove if $A$ is a trivial antichain. Suppose that $A$ is nontrivial. The relations

$$
\mathfrak{b}_{k}^{X}(A) \backslash_{k} X \leq \mathfrak{b}_{k}^{X}(A) \leq \mathfrak{b}_{k}^{X}(A) /_{k} X, \quad \mathfrak{b}_{k}^{X}\left(A /{ }_{k} X\right) \leq \mathfrak{b}_{k}^{X}(A) \leq \mathfrak{b}_{k}^{X}\left(A \backslash_{k} X\right)
$$

follow from Lemmas 3.3 and 2.4(ii).

We need the following auxiliary relations. If $A^{\prime}$ and $A^{\prime \prime}$ are arbitrary antichains of $P$, then

$$
\begin{aligned}
& \left(A^{\prime} \wedge A^{\prime \prime}\right) \backslash_{k} X \leq\left(A^{\prime} \backslash_{k} X\right) \wedge\left(A^{\prime \prime} \backslash_{k} X\right), \\
& \left(A^{\prime} \wedge A^{\prime \prime}\right) /_{k} X \leq\left(A^{\prime} /_{k} X\right) \wedge\left(A^{\prime \prime} /_{k} X\right) .
\end{aligned}
$$

To prove $\mathfrak{b}_{k}^{X}(A) \backslash_{k} X \leq \mathfrak{b}_{k}^{X}\left(A /{ }_{k} X\right)$, we use (3.17) and (3.11), and we see that

$$
\begin{aligned}
\mathfrak{b}_{k}^{X}(A) \backslash_{k} X & =\left(\bigwedge_{a \in A} \mathfrak{b}_{k}^{X}(a)\right) \backslash_{k} X \leq \bigwedge_{a \in A}\left(\mathfrak{b}_{k}^{X}(a) \backslash_{k} X\right) \leq \bigwedge_{a \in A} \mathfrak{b}_{k}^{X}\left(\{a\} /{ }_{k} X\right) \\
& =\mathfrak{b}_{k}^{X}\left(\bigvee_{a \in A}\left(\{a\} /_{k} X\right)\right)=\mathfrak{b}_{k}^{X}\left(A / /_{k} X\right) .
\end{aligned}
$$


To prove $\mathfrak{b}_{k}^{X}(A) /_{k} X \leq \mathfrak{b}_{k}^{X}\left(A \backslash_{k} X\right)$, we use (3.18) and (3.11), and we see that

$$
\begin{aligned}
\mathfrak{b}_{k}^{X}(A) /_{k} X & =\left(\bigwedge_{a \in A} \mathfrak{b}_{k}^{X}(a)\right) /_{k} X \leq \bigwedge_{a \in A}\left(\mathfrak{b}_{k}^{X}(a) /_{k} X\right) \leq \bigwedge_{a \in A} \mathfrak{b}_{k}^{X}\left(\{a\} \backslash_{k} X\right) \\
& =\mathfrak{b}_{k}^{X}\left(\bigvee_{a \in A}\left(\{a\} \backslash_{k} X\right)\right)=\mathfrak{b}_{k}^{X}\left(A \backslash_{k} X\right)
\end{aligned}
$$

\section{REFERENCES}

[1] M. Aigner, Combinatorial Theory, Grundlehren der Mathematischen Wissenschaften, vol. 234, Springer-Verlag, Berlin, 1979.

[2] J. Edmonds and D. R. Fulkerson, Bottleneck extrema, J. Combinatorial Theory 8 (1970), 299-306.

[3] A. Lehman, A solution of the Shannon switching game, J. Soc. Indust. Appl. Math. 12 (1964), 687-725.

[4] A. O. Matveev, On blockers in bounded posets, Int. J. Math. Math. Sci. 26 (2001), no. $10,581-588$.

[5] _ A note on operators of deletion and contraction for antichains, Int. J. Math. Math. Sci. 31 (2002), no. 12, 725-729.

[6] P. D. Seymour, The forbidden minors of binary clutters, J. London Math. Soc. (2) 12 (1975/1976), no. 3, 356-360.

[7] R. P. Stanley, Enumerative Combinatorics. Vol. 1, Cambridge Studies in Advanced Mathematics, vol. 49, Cambridge University Press, Cambridge, 1997.

Andrey O. Matveev: Data-Center Company, RU-620034, P.O. Box 5, Ekaterinburg, Russia

E-mail address: aomatveev@dc.ru 


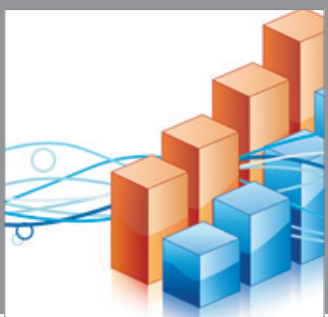

Advances in

Operations Research

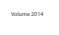

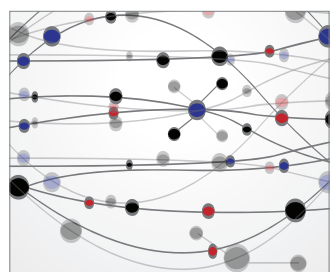

\section{The Scientific} World Journal
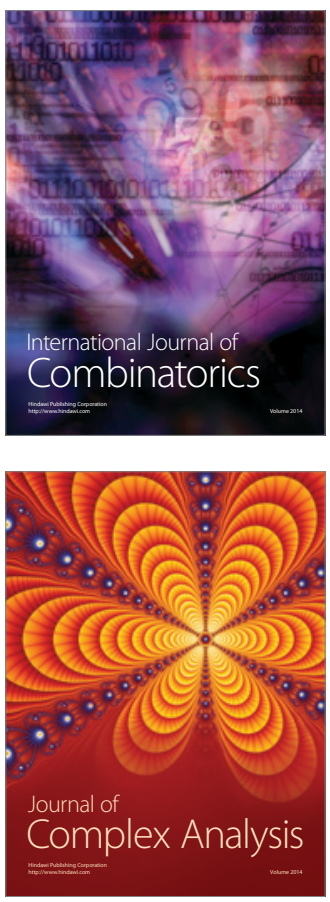

International Journal of

Mathematics and

Mathematical

Sciences
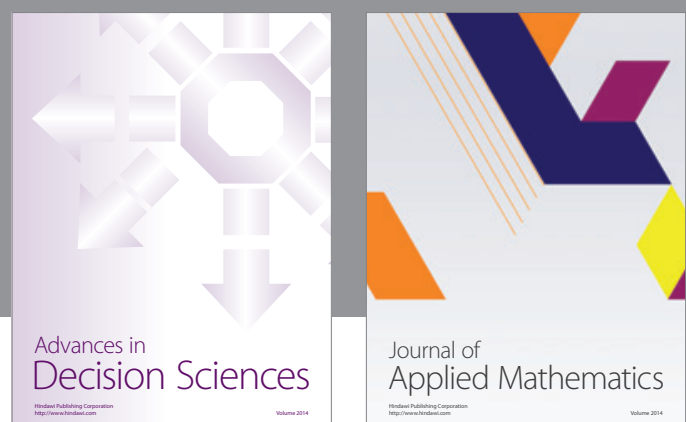

Journal of

Applied Mathematics
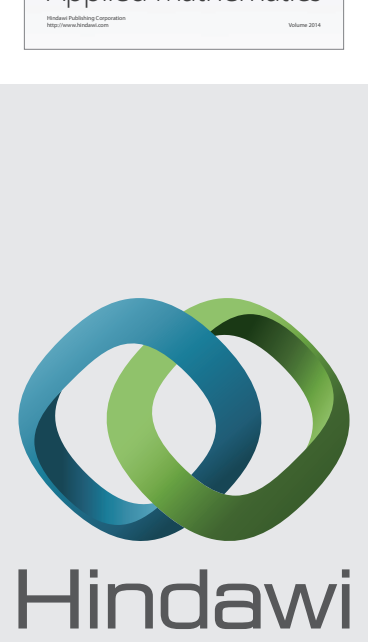

Submit your manuscripts at http://www.hindawi.com
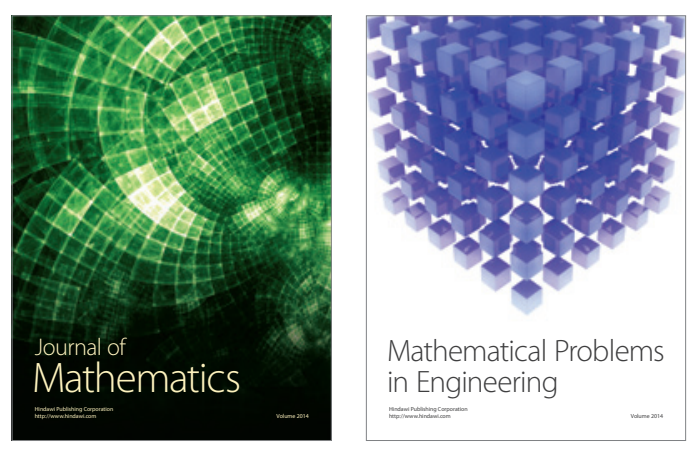

Mathematical Problems in Engineering
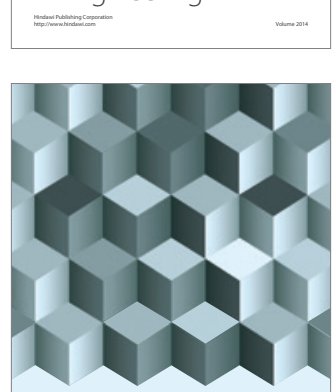

Journal of

Function Spaces
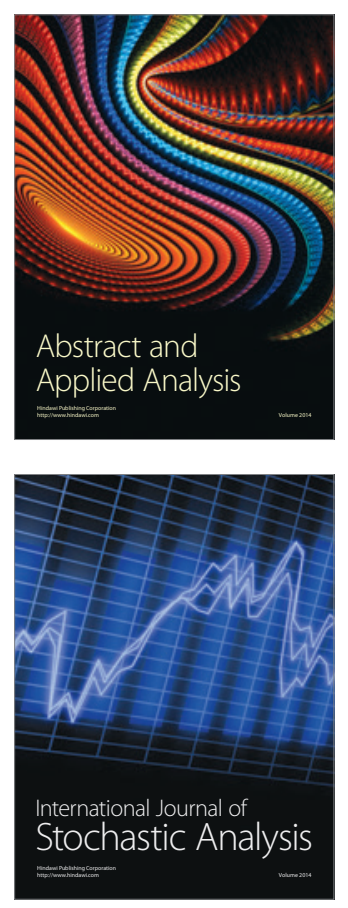

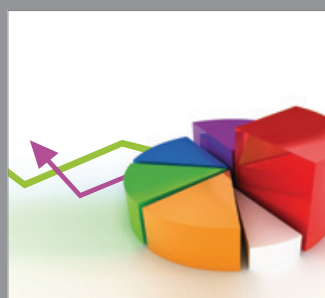

ournal of

Probability and Statistics

Promensencen
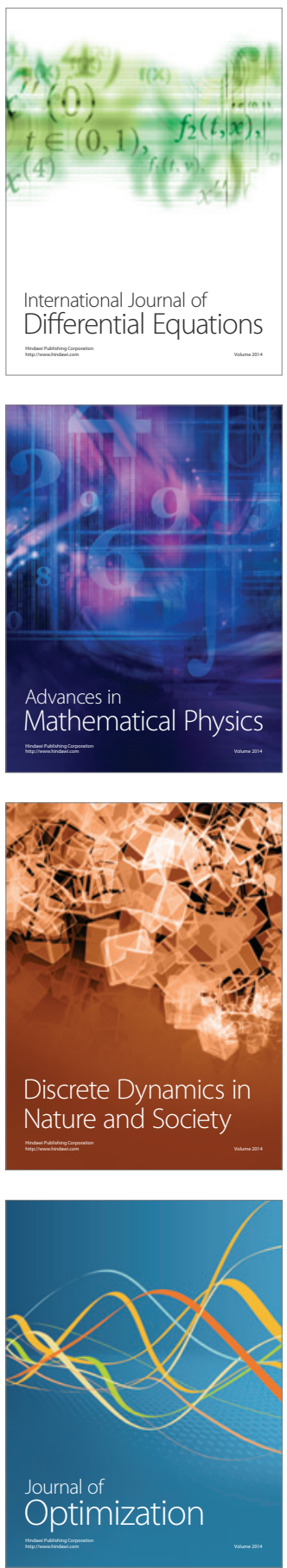